\title{
Simultaneous Embeddability of Two Partitions
}

\author{
Jan Christoph Athenstädt ${ }^{1}$, Tanja Hartmann ${ }^{2}$, and Martin Nöllenburg ${ }^{2}$ \\ 1 Department of Computer and Information Science, University of Konstanz, Germany \\ 2 Institute of Theoretical Informatics, Karlsruhe Institute of Technology (KIT), Germany
}

\begin{abstract}
We study the simultaneous embeddability of a pair of partitions of the same underlying set into disjoint blocks. Each element of the set is mapped to a point in the plane and each block of either of the two partitions is mapped to a region that contains exactly those points that belong to the elements in the block and that is bounded by a simple closed curve. We establish three main classes of simultaneous embeddability (weak, strong, and full embeddability) that differ by increasingly strict well-formedness conditions on how different block regions are allowed to intersect. We show that these simultaneous embeddability classes are closely related to different planarity concepts of hypergraphs. For each embeddability class we give a full characterization. We show that (i) every pair of partitions has a weak simultaneous embedding, (ii) it is NP-complete to decide the existence of a strong simultaneous embedding, and (iii) the existence of a full simultaneous embedding can be tested in linear time.
\end{abstract}

\section{Introduction}

Pairs of partitions of a given set of objects occur naturally when evaluating two alternative clusterings in the field of data analysis and data mining. A clustering partitions a set of objects into blocks or clusters, such that objects in the same cluster are more similar (according to some notion of similarity) than objects in different clusters. There are a multitude of clustering algorithms that use, e.g., an underlying graph structure or an attribute-based distance measure to define similarities. Many algorithms also provide configurable parameter settings. Consequently, different algorithms return different clusterings and judging which clustering is the most meaningful with respect to a certain interpretation of the data must be done by a human expert. For a structural comparison of two clusterings several numeric measures exist [20], however, a single numeric value hardly shows where the clusterings agree or disagree. Hence, a data analyst may want to compare different clusterings visually, which motivates the study of simultaneous embeddability of two partitions.

We provide fundamental characterizations and complexity results regarding the simultaneous embeddability of a pair of partitions. While simultaneous embeddability can generally be defined for any number $k \geq 2$ of partitions, we focus on the basic case of embedding two partitions, which is also the most relevant one in the data analysis application. We propose to embed two alternative partitions of the same set $U$ into the plane $\mathbb{R}^{2}$ by mapping each element of $U$ to a unique point and each block (of either of the two partitions) to a region bounded by a simple closed curve. Each block region must contain all points that belong to elements in that block and no point whose element belongs to a different block. Hence, in total, each point lies inside two block regions.

C. Duncan and A. Symvonis (Eds.): GD 2014, LNCS 8871, pp. 64 75 2014.

(c) Springer-Verlag Berlin Heidelberg 2014 
A simultaneous embedding of two partitions shares certain properties with set visualizations like Euler or Venn diagrams [8 12,19]. Its readability will be affected by wellformedness conditions for the intersections of the different block regions. Accordingly, we define a (strict) hierarchy of embeddability classes based on increasingly tight wellformedness conditions: weak, strong, and full embeddability. We show that (i) any two partitions are weakly embeddable, (ii) the decision problem for strong embeddability is NP-complete, and (iii) there is a linear-time decision algorithm for full embeddability. We fully characterize the embeddability classes in terms of the existence of a planar support (strong embeddability) or in terms of the planarity of the bipartite map (full embeddability). Interestingly, both concepts are closely related to hypergraph embeddings and different notions of hypergraph planarity. Our NP-completeness result implies that vertex-planarity testing of 2-regular hypergraphs is also NP-complete.

\subsection{Related Work}

In information visualization there are a large variety of techniques for visualizing clusters of objects, some of which simply map objects to (colored) points so that spatial proximity indicates object similarity [6, 16], others explicitly visualize clusters or general sets as regions in the plane [9],19]. These approaches are visually similar to Euler diagrams [8, 12], however, they do not give hard guarantees on the final set layout, e.g., in terms of intersection regions or connectedness of regions, nor do they specifically consider the simultaneous embedding of two or more clusterings or partitions.

Clustered planarity is a concept in graph drawing that combines a planar graph layout with a drawing of the clusters of a single hierarchical clustering. Clusters are represented as regions bounded by simple closed and pairwise crossing-free curves. Such a layout is called $c$-planar if no edge crosses a region boundary more than once [11].

The simultaneous embedding of two planar graphs on the same vertex set is a topic that is well studied in the graph drawing literature, see the recent survey of Bläsius et al. [2]. In a simultaneous graph embedding each vertex is located at a unique position and edges contained in both graphs are represented by the same curve for both graphs. The remaining (non-shared) edges are embedded so that each graph layout by itself is crossing-free, but edges from the first graph may cross edges in the second graph.

Some of our results and concepts in this paper can be seen as a generalization of simultaneous graph embedding to simultaneous hypergraph embedding if we consider blocks as hyperedges: all vertices are mapped to unique points in the plane and two hyperedges, represented as regions bounded by simple closed curves, may only intersect if they belong to different hypergraphs or if they share common vertices. Several concepts for visualizing a single hypergraph are known [4, 5, 14, 15, 17], but to the best of our knowledge the simultaneous layout of two or more hypergraphs has not been studied.

\subsection{Preliminaries}

Let $U=\left\{u_{1}, \ldots, u_{m}\right\}$ be a finite universe. A partition $\mathcal{P}=\left\{B_{1}, \ldots, B_{n}\right\}$ of $U$ groups the elements of $U$ into disjoint blocks, i.e., every element $u \in U$ is contained in exactly one block $B_{i} \in \mathcal{P}$. In this paper, we consider pairs $\left\{\mathcal{P}_{0}, \mathcal{P}_{1}\right\}$ of partitions of the same 


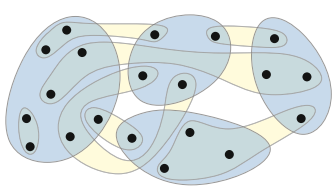

(a) weak embedding

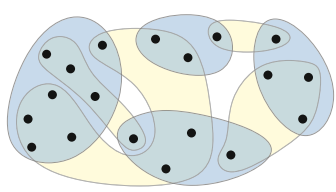

(b) strong embedding

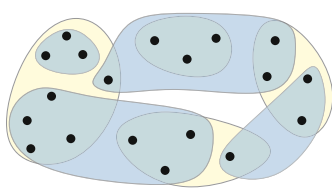

(c) full embedding

Fig. 1. Examples of simultaneous embeddings of two partitions

universe $U$, i.e., each element $u \in U$ is contained in one block of $\mathcal{P}_{0}$ and in one block of $\mathcal{P}_{1}$. In the following we often omit to mention $U$ explicitly.

Let $\mathcal{S}$ be a collection of subsets of $U$. An embedding $\Gamma$ of $\mathcal{S}$ maps every element $u \in U$ to a distinct point $\Gamma(u) \in \mathbb{R}^{2}$ and every set $S \in \mathcal{S}$ to a simple, bounded, and closed region $\Gamma(S) \subset \mathbb{R}^{2}$ such that $\Gamma(u) \in \Gamma(S)$ if and only if $u \in S$. Moreover, we require that each contiguous intersection between the boundaries of two regions is in fact a crossing point $p \in \mathbb{R}^{2}$, i.e., the local cyclic order of the boundaries alternates around $p$. A simultaneous embedding $\Gamma$ of a pair of partitions $\left\{\mathcal{P}_{0}, \mathcal{P}_{1}\right\}$ is an embedding of the union $\mathcal{P}_{0} \cup \mathcal{P}_{1}$ of the two partitions. We define $R_{B}=\Gamma(B)$ as the block region of a block $B$ and denote its boundary by $\partial R_{B}$. Figure 1 shows examples of simultaneous embeddings in the three different embedding classes to be defined in Section 2

A simultaneous embedding $\Gamma$ induces a subdivision of the plane and we can derive a plane multigraph $G_{\Gamma}$ by introducing a node for each intersection of two boundaries and an edge for each section of a boundary that lies between two intersections. Furthermore, a boundary without intersections is replaced by a node with a self loop nested inside its surrounding face. We call $G_{\Gamma}$ the contour graph of $\Gamma$ and its dual graph $G_{\Gamma}^{*}$ the dual graph of $\Gamma$. The faces of $G_{\Gamma}$ belong to zero, one, or two block regions. We call a face that belongs to no block region a background face, a face that belongs to a single block region a linking face, and a face that belongs to two block regions an intersection face. Only intersection faces contain points corresponding to elements in the universe, and no two faces of the same type are adjacent in the contour graph.

Alternatively, the union of the two partitions $\mathcal{P}_{0} \cup \mathcal{P}_{1}$ can also be seen as a hypergraph $H=\left(U, \mathcal{P}_{0} \cup \mathcal{P}_{1}\right)$, where every element $u \in U$ is a vertex and every block defines a hyperedge, i.e., a non-empty subset of $U$. The hypergraph $H$ is 2-regular since every vertex is contained in exactly two hyperedges. We denote $H=H\left(\mathcal{P}_{0}, \mathcal{P}_{1}\right)$ as the corresponding hypergraph of the pair of partitions $\left\{\mathcal{P}_{0}, \mathcal{P}_{1}\right\}$.

Hypergraph supports [15] play an important role in hypergraph embeddings and their planarity. A support of a hypergraph $H=(V, \mathcal{S})$ is a graph $G_{p}=(V, E)$ on the vertices of $H$, such that the induced subgraph $G_{p}[S]$ of every hyperedge $S \in \mathcal{S}$ is connected. We extend the concept of supports to pairs of partitions, i.e., we say that a graph $G_{p}=(V, E)$ is a support for $\left\{\mathcal{P}_{0}, \mathcal{P}_{1}\right\}$, if it is a support of $H\left(\mathcal{P}_{0}, \mathcal{P}_{1}\right)$.

We call a support path based, if the induced subgraphs of all hyperedges are paths 1 and tree based, if all hyperedge-induced subgraphs are trees, i.e., they do not contain

\footnotetext{
${ }^{1}$ Brandes et al. [4] used a slightly different definition and called a support path based if the induced subgraph of each hyperedge has a Hamiltonian path.
} 
any cycles. For any support $G_{p}$ of a pair of partitions $\left\{\mathcal{P}_{0}, \mathcal{P}_{1}\right\}$ we can always create a tree-based support $G_{p}^{\prime}$ by removing edges from cycles: Suppose there exists a block $B \in \mathcal{P}_{0}$ such that $G_{p}[B]$ contains a cycle $K$. If the vertices in $K$ are also contained in a common block of $\mathcal{P}_{1}$, we can just remove a random edge from $K$ without destroying the support property. Otherwise, we can remove an edge from $K$ that connects vertices in two different blocks of $\mathcal{P}_{1}$ without destroying the support property.

The bipartite map $G_{b}(H)$ of a hypergraph $H=(V, \mathcal{S})$ is defined as the bipartite graph $G_{b}(H)=\left(V \cup \mathcal{S}, E_{b}\right)$ that has a node for each vertex in $V$ and for each hyperedge in $\mathcal{S}$ [21]. A node $v \in V$ is adjacent to a node $S \in \mathcal{S}$ if $v \in S$. We say that $G_{b}(H)$ is the bipartite map of a pair of partitions $\left\{\mathcal{P}_{0}, \mathcal{P}_{1}\right\}$ if $H=H\left(\mathcal{P}_{0}, \mathcal{P}_{1}\right)$.

Finally, we define the block intersection graph $G_{s}\left(\mathcal{P}_{0}, \mathcal{P}_{1}\right)$ as the graph with vertex set $V_{s}=\mathcal{P}_{0} \cup \mathcal{P}_{1}$ and edge set $E_{s}=\left\{\left\{B, B^{\prime}\right\} \mid B \cap B^{\prime} \neq \emptyset\right\}$. Thus $G_{s}$ has a vertex for each block and an edge between any two blocks that share a common element. Since only blocks of different partitions can intersect, we know that $G_{s}$ is bipartite.

\section{The Main Classes of Embeddability}

We define three main concepts of simultaneous embeddability for pairs of partitions. We will see that these concepts induce a hierarchy of embeddability classes of pairs of partitions. We begin with weak embeddability, which is the most general concept.

Definition 1 (Weak Embeddability). A simultaneous embedding of two partitions is weak if no two block regions of the same partition intersect. Two partitions are weakly embeddable if they have a weak simultaneous embedding.

Prohibiting intersections of block regions of the same partition is our first well-formedness condition. A weak embedding emphasizes the fact that the blocks in each partition are disjoint. Since the blocks of any partition are disjoint by definition, it is not surprising that any pair of partitions is weakly embeddable (see Fig. 1(a) for an example).

Theorem 1. Any two partitions of a common universe are weakly embeddable on any point set.

Proof. A spanning forest (in fact, any planar graph) on $n$ nodes can always be drawn in a planar way on any fixed set of $n$ points in the plane [18]. Let now $\mathcal{P}$ be a partition. We choose arbitrary, but distinct points in the plane for the elements of $U$. We then generate a spanning tree on the elements in each block and embed the resulting forest in a planar way on the points. Slightly inflating the thickness of the edges of the trees yields simple bounded block regions. We can do this independently for a second partition on the same points and obtain a weak simultaneous embedding.

Although the concept of weak embedding does not seem to provide interesting insights into the structure of a given pair of partitions, it guarantees at least the existence of a simultaneous embedding for any pair of partitions that is more meaningful than an arbitrary embedding. An obvious drawback of weak embeddings is that the block regions of disjoint blocks are allowed to intersect, as long as both blocks belong to different partitions - even if they do not share common elements. Following the general idea of Euler diagrams [8], which do not show regions corresponding to empty 
intersections, we establish a stricter concept of embeddability. In a strong embedding block regions may only intersect if the corresponding blocks have at least one element in common, and even more, each intersection face of the contour graph must actually contain a point, see Fig. 1(b)] This is our second well-formedness condition.

Definition 2 (Strong Embeddability). A simultaneous embedding $\Gamma$ of two partitions is strong if each intersection face of the corresponding contour graph contains a point $\Gamma(u)$ for some $u \in U$. Two partitions are strongly embeddable if they have a strong simultaneous embedding.

Obviously, a strong embedding is also weak, since blocks of the same partition have no common elements, and thus, cannot form intersection faces. The class of strongly embeddable pairs of partitions is characterized by Theorem 2, we show in Section 3 that deciding the strong embeddability of a pair of partitions is NP-complete.

Theorem 2. A pair of partitions of a common universe is strongly embeddable if and only if it has a planar support.

Proof. Let $\left\{\mathcal{P}_{0}, \mathcal{P}_{1}\right\}$ be a pair of partitions and let $G_{\Gamma}$ be the contour graph resulting from a strong embedding $\Gamma$ of $\left\{\mathcal{P}_{0}, \mathcal{P}_{1}\right\}$. We construct a planar support of $\left\{\mathcal{P}_{0}, \mathcal{P}_{1}\right\}$ along $G_{\Gamma}$ as follows. First recall that the elements of the universe, which correspond to the nodes in a support, are represented in $\Gamma$ by points that are drawn inside intersection faces. Vice versa, since $\Gamma$ is strong, each intersection face contains at least one point. Hence, we choose one point in each intersection face as the center of this face. We now create a dummy vertex for each linking face (observe that one block region may induce several linking faces) and link it to the centers of all adjacent intersection faces. The resulting graph is a subgraph of the dual graph of the contour graph $G_{\Gamma}$ and therefore planar. We now connect all remaining vertices in a star-like fashion to the center of their intersection face, routing the edges in a non-crossing way. We finally remove the dummy vertices by merging them to an adjacent center, linking all adjacent vertices to that center. This graph remains planar. It also has the support property, since all intersection and linking faces of any block region are connected into a single component, and with them all vertices of that block region.

Now we construct a strong embedding from a planarly embedded support of $\left\{\mathcal{P}_{0}, \mathcal{P}_{1}\right\}$. To this end, we first construct a tree-based support by deleting edges from cycles as described in Section 1.2. Then, we simply inflate the edges of each block-induced subtree. Since the underlying support is embedded in a planar way, this yields a simple block region for every block in $\left\{\mathcal{P}_{0}, \mathcal{P}_{1}\right\}$ such that two block regions only intersect at the positions of the nodes. Hence, the constructed block regions together with the nodes of the support form a strong embedding of $\left\{\mathcal{P}_{0}, \mathcal{P}_{1}\right\}$. We note that the support graph as a planar graph can in fact be embedded on any point set [18]. Hence, a strongly embeddable pair of partitions can be strongly embedded on any point set.

In a strong embedding, a single block region may still cross other block regions and intersect the same block regions several times forming distinct intersection faces-as long as each intersection face contains at least one common point. The last of our three embeddability classes prevents this behavior and requires that the block regions form a collection of pseudo-disks, i.e., the boundaries of every pair of regions intersect at most 
twice and the boundaries of two nested regions do not intersect. See Fig 1(c) for an example. This implies in particular that every block intersection is connected, which is a well-formedness condition widely used in the context of Euler diagrams [8], and that block regions do not cross and are thus more locally confined.

Definition 3 (Full Embeddability). A simultaneous embedding of two partitions is full if it is a strong embedding and the regions form a collection of pseudo-disks. Two partitions are fully embeddable if they have a full simultaneous embedding.

Using a linear-time algorithm for planarity testing [13], the following characterization of fully embeddable pairs of partitions directly implies a linear-time algorithm for deciding full embeddability. The proof of Theorem 3 constructs a bipartite map along a given full embedding, and vice versa. It uses similar techniques as the proofs of Theorems 1 and 2 and is found in the full version of this paper [1].

Theorem 3. A pair of partitions of a common universe is fully embeddable if and only if its bipartite map is planar.

A full embedding is strong by definition and we have seen above that a strong embedding is also weak. Hence, the three embeddability classes introduced in this section induce a hierarchy of embeddability classes. In the full version [1] we show that this hierarchy is strict. The weak embeddability class forms the basis of the hierarchy and contains all pairs of partitions. The strong embeddability class and the full embeddability class are characterized by the existence of a planar support and the planarity of the bipartite map of a pair of partitions, respectively, where the latter directly implies a linear time algorithm for the corresponding decision problem. Moreover, these characterizations reveal close relations to the hypergraph planarity concepts of Zykov and vertex planarity.

A hypergraph $H=(V, \mathcal{S})$ is Zykov-planar [22], if there exists a subdivision of the plane into faces, such that each hyperedge $S \in \mathcal{S}$ can be mapped to a face of the subdivision, and each vertex $v \in V$ can be mapped to a point on the boundary of all faces that represent a hyperedge containing $v$. Walsh [21] showed that a hypergraph is Zykov planar if and only if its bipartite map is planar.

In contrast, a hypergraph $H=(V, \mathcal{S})$ is vertex-planar [14] if there exists a subdivision of the plane into faces, such that every vertex $v \in V$ can be mapped to a face and for every hyperedge $S \in \mathcal{S}$, the interior of the union of all faces of the vertices in $S$ is connected. Kaufmann et al. [15] showed that a hypergraph is vertex planar if and only if it has a planar support. This shows that the class of fully embeddable pairs of partitions is a subclass of Zykov planar hypergraphs, and the class of strongly embeddable pairs of partitions is a subclass of vertex planar hypergraphs.

\section{Complexity of Deciding Strong Embeddability}

In this section we show the NP-completeness of testing strong embeddability. As a consequence, testing whether the corresponding hypergraph of a pair of partitions has a planar support is also NP-complete by Theorem 2. This seems not very surprising considering the more general hardness results of Johnson and Pollak [14] and Buchin et 


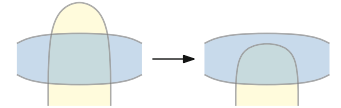

(a) Removing a linking face

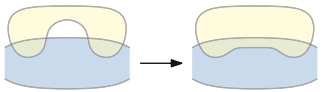

(b) Removing a background face

Fig. 2. Two cases for transforming a strong embedding into a proper strong embedding

al. [5] who showed that deciding the existence of a planar support and a 2-outerplanar support in general hypergraphs is NP-hard. However, we consider a restricted subclass of 2-regular hypergraphs, thus, the NP-hardness of our problem does not directly follow from the previous results. Moreover, other special cases, e.g., finding path, cycle, tree, and cactus supports are known to be solvable in polynomial time [3, 5, 14]. Together with the characterization of Theorem 2 , Theorem 4 immediately implies that testing the vertex planarity of a 2-regular hypergraph is NP-complete.

Theorem 4. Deciding the strong embeddability of a pair of partitions is NP-complete.

The existing hardness results [5, 14] rely on elements that are contained in more than two hyperedges and could not be adapted to our 2-regular setting. Instead we prove the hardness of deciding strong embeddability by a quite different reduction from the NPcomplete problem MONOTONE PLANAR 3SAT [10]. A monotone planar 3Sat formula $\varphi$ is a 3Sat formula whose clauses either contain only positive or only negated literals (we call these clauses positive and negative) and whose variable-clause graph $H_{\varphi}$ is planar. A monotone rectilinear representation (MRR) of $\varphi$ is a drawing of $H_{\varphi}$ such that the variables correspond to axis-aligned rectangles on the $\mathrm{x}$-axis and clauses correspond to non-crossing E-shaped "combs" above the x-axis if they contain only positive variables and below the X-axis otherwise; see Fig. 4(a)

An instance of MONOTONE PLANAR 3SAT is an MRR of a monotone planar 3Sat formula $\varphi$. In the proof of Theorem 4 we will construct a pair of partitions $\left\{\mathcal{P}_{0}, \mathcal{P}_{1}\right\}_{\varphi}$ that admits a strong embedding if and only if $\varphi$ is satisfiable.

For the sake of simplicity, we restrict the class of strong embeddings to the subclass of proper strong embeddings, which is equivalent, as we can argue that a pair of partitions has a strong embedding if and only if it also has a proper one. A strong embedding is proper if the contour graph does not contain background or linking faces that are adjacent to only two other faces. Figure 2 illustrates how background or linking faces violating this condition can be removed, transforming a strong embedding into a proper one. We say that two proper strong embeddings are equivalent if the embeddings of their contour graphs are equivalent, i.e. if the cyclic order of the edges around each vertex is the same. A pair of partitions has a unique strong embedding if all proper strong embeddings are equivalent. Note that, analogously to the definition of equivalence of planar graph embeddings, two equivalent proper strong embeddings may have different unbounded outer background faces. Our construction in the hardness proof is independent of the choice of the outer face.

Next we define a special pair of partitions that has a unique grid-shaped embedding as a scaffold for the gadgets in the subsequent proof of Theorem 4 . The first step is 

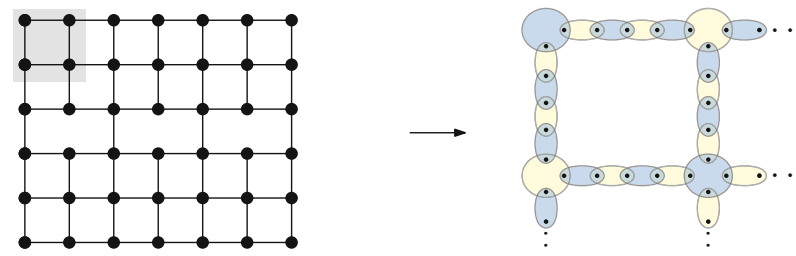

$\mathcal{Q}_{0}$

$\mathcal{Q}_{1}$

Fig. 3. Graph $G_{2,3}$ and the partitions $\left\{\mathcal{Q}_{0}, \mathcal{Q}_{1}\right\}$ sketched for the top-left grid cell marked in gray

to construct a base graph $G_{m, n}$ for two integers $m$ and $n$. The graph $G_{m, n}$ is a grid with $m n+1$ columns and $2 m+2$ rows of vertices with integer coordinates $(i, j)$ for $0 \leq i \leq m n$ and $0 \leq j \leq 2 m+1$. Each vertex $v$ with coordinates $(i, j)$ is connected to the four vertices at coordinates $(i-1, j),(i+1, j),(i, j-1),(i, j+1)$ (if they exist). Between the middle rows $m$ and $m+1$ we remove all vertical edges except for those in columns $0, m, 2 m, \ldots, n m$. This defines $n$ larger grid cells of width $m$ in this particular row. Figure 3 (left) shows an example.

From $G_{m, n}$ we construct a pair of partitions $\left\{\mathcal{Q}_{0}, \mathcal{Q}_{1}\right\}$ as follows (see Fig. 3). For each vertex $v$ with coordinates $(i, j)$ we create a vertex block $B_{v}$ in partition $\mathcal{Q}_{(i+j)(\bmod 2)}$. For each edge $(u, v)$ in $G_{m, n}$ we create a chain of four edge blocks $B_{u, v}^{1}, B_{u, v}^{2}, B_{u, v}^{3}, B_{u, v}^{4}$, such that $B_{u, v}^{1}$ and $B_{u, v}^{3}$ are in the same partition as $B_{v}$ and $B_{u, v}^{2}$ and $B_{u, v}^{4}$ are in the same partition as $B_{u}$. We distribute five distinct elements among the edge blocks of $(u, v)$ and the vertex blocks for $u$ and $v$ such that they form the desired chain pattern and each intersection face contains one common element. The pair $\left\{\mathcal{Q}_{0}, \mathcal{Q}_{1}\right\}$ is indeed a pair of partitions as every element belongs to exactly one block of each partition. Edge blocks contain two and vertex blocks up to four elements (depending on the degree of the corresponding vertex in $G_{m, n}$ ). Below we will add the gadgets of the reduction on top of $\left\{\mathcal{Q}_{0}, \mathcal{Q}_{1}\right\}$, for which it is required that there is an edge block in each partition that does not share any element with a vertex block. This explains why we link blocks of adjacent vertices by chains of four blocks.

The next lemma shows that $\left\{\mathcal{Q}_{0}, \mathcal{Q}_{1}\right\}$ has a unique embedding (proof in the full version [1]), which is a consequence of the fact that $G_{m, n}$ is a subdivision of a planar 3 -connected graph (assuming $n \geq 2$ ) and thus it has a unique embedding. This property is inherited by $\left\{\mathcal{Q}_{0}, \mathcal{Q}_{1}\right\}$ in our construction.

Lemma 1. The pair of partitions $\left\{\mathcal{Q}_{0}, \mathcal{Q}_{1}\right\}$ has a unique embedding.

Now we have all the tools that we need to prove our main theorem in this section.

Proof (of Theorem 4). First we show that the problem is in NP. By Theorem 2 we know that a pair of partitions is strongly embeddable if and only if it has a planar support. Thus we can "guess" a graph on $U$ and then test its planarity and support property in polynomial time. This shows membership in NP. It remains to describe the hardness reduction.

Let $\varphi$ be a planar monotone 3Sat formula together with an MRR. First we construct the pair of partitions $\left\{\mathcal{Q}_{0}, \mathcal{Q}_{1}\right\}$ for the base graph $G_{m, n}$, where $m$ is the number of clauses of $\varphi$ and $n$ is the number of variables of $\varphi$. By Lemma $1\left\{\mathcal{Q}_{0}, \mathcal{Q}_{1}\right\}$ has a unique 


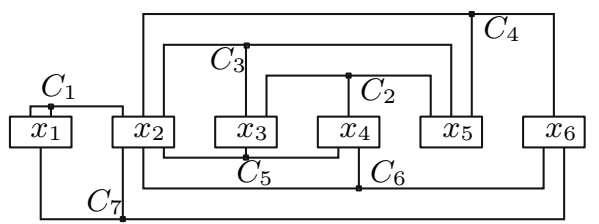

$$
\left.\begin{array}{l}
C_{1}=\left(x_{1} \vee x_{1} \vee x_{2}\right) \\
C_{2}=\left(x_{2} \vee x_{3} \vee x_{5}\right) \\
C_{3}=\left(x_{3} \vee x_{4} \vee x_{5}\right) \\
C_{4}=\left(x_{2} \vee x_{5} \vee x_{6}\right. \\
C_{5}=\left(\overline{x_{2}} \vee \overline{x_{3}} \vee \overline{x_{4}}\right. \\
C_{6}=\left(\overline{x_{2}} \vee \overline{x_{4}} \vee \overline{x_{6}}\right. \\
C_{7}=\left(\overline{x_{1}} \vee \overline{x_{2}} \vee \overline{x_{6}}\right)
\end{array}\right)
$$

(a) Monotone rectilinear representation of a formula $\varphi$

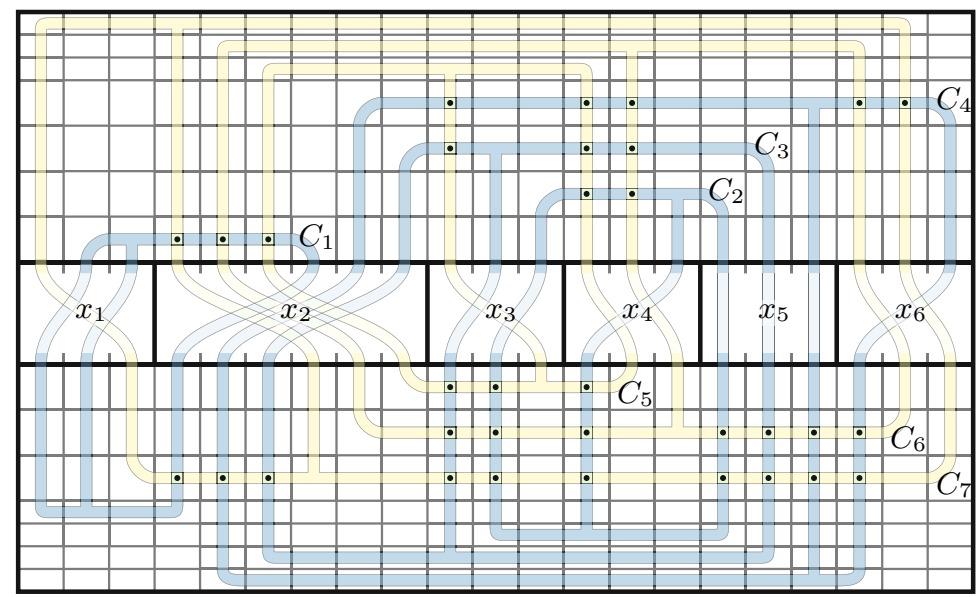

(b) Sketch of the clause blocks laid on top of the grid $\left\{\mathcal{Q}_{0}, \mathcal{Q}_{1}\right\}$ (empty columns omitted)

Fig. 4. Illustration of the NP-hardness reduction

proper grid-like embedding. We call $\left\{\mathcal{Q}_{0}, \mathcal{Q}_{1}\right\}$ the base grid and the $n$ special cells between rows $m$ and $m+1$ the variable cells of the base grid.

Next we augment the pair of partitions $\left\{\mathcal{Q}_{0}, \mathcal{Q}_{1}\right\}$ by additional blocks, one for each clause, where positive clauses are added to $\mathcal{Q}_{0}$ and negative clauses to $\mathcal{Q}_{1}$. The definition of these clause blocks closely follows the layout of the given MRR, see Fig. 4(a), Let $C_{1}, C_{2}, \ldots, C_{l}$ be the positive clauses of $\varphi$ ordered so that if $C_{i}$ is nested inside the E-shape of $C_{j}$ in the given MRR then $i<j$. Analogously let $C_{l+1}, \ldots, C_{m}$ be the ordered negative clauses. We describe the definition of the block $B_{i}$ for a positive clause $C_{i}(1 \leq i \leq l)$; blocks for negative clauses are defined symmetrically. We create an intermediate embedding of $B_{i}$ (which is not yet strong but serves as a template for a later strong embedding) by putting $B_{i}$ on top of the base grid 2 and adding new elements to $B_{i}$ and to certain edge blocks in $\mathcal{Q}_{1}$. This fixes $B_{i}$ to run through two mirrored E-shaped sets of grid cells of our choice (Fig. 4(b)). In the upper half of the base grid, $B_{i}$ is assigned to run between rows $m-i$ and $m-i+1$. Furthermore, $B_{i}$ is assigned to three columns leading towards the variable cells from the top. Let $x_{j}$ be a variable contained in $C_{i}$ and assume that $C_{i}$ is the $k$-th positive clause from the right connecting to $x_{j}$ in the embedding of the given MRR. Then $B_{i}$ runs between columns $j m-k$ and

\footnotetext{
${ }^{2}$ The idea of fixing paths to an underlying grid is inspired by Chaplick et al. [7].
} 
$j m-k+1$. In the lower half of the base grid we translate and mirror the resulting E-shape as follows. We let $B_{i}$ occupy the cells between rows $2 m+2-l+i-1$ and $2 m+2-l+i$ and the three columns are shifted to the left by the number of occurrences of the respective variable in negative clauses (Fig. 4(b)). Since each variable cell is $m$ columns wide, we can always assign each clause to a unique column of $x_{j}$ in the top and bottom half of the grid in this way.

We actually fix $B_{i}$ to the base grid by adding one shared element for each crossed edge of a grid cell to both $B_{i}$ and the respective edge block of $\mathcal{Q}_{1}$ that does not share an element with a vertex block in $\mathcal{Q}_{0}$ (recall that $\left\{\mathcal{Q}_{0}, \mathcal{Q}_{1}\right\}$ contains such a block in each partition and for each grid edge). No two blocks of the same clause type (positive or negative) intersect, but blocks of different type do intersect in certain grid cells. For each grid cell shared between a positive and negative block (except for the $n$ variable cells) we add one shared element (black dots in Fig. 4(b) and call the respective grid cell the home cell for this element. Recall that the orders of the incoming blocks from the top and the bottom of each variable cell are inverted. Thus, within each variable cell the blocks of each pair of a positive and negative clause using the corresponding variable intersect, but no shared element is added. We denote the resulting new pair of partitions as $\left\{\mathcal{P}_{0}, \mathcal{P}_{1}\right\}_{\varphi}$ and observe that its size is polynomial in the size of $\varphi$.

Next we argue about the strong embedding options in contrast to the immediate embedding for a clause block $B_{i}$ in $\left\{\mathcal{P}_{0}, \mathcal{P}_{1}\right\}_{\varphi}$. In the intermediate embedding each block has three connections through variable cells linking the upper E-shape with the lower E-shape. Any element shared with an edge block of the uniquely embedded base grid must obviously be reached by the block region of $B_{i}$. Since the block region must be simple, any strong embedding of $B_{i}$ results from opening the intermediate embedding of $B_{i}$ in exactly two grid cells so that the resulting block region of $B_{i}$ is connected and has no holes. Additionally, a shared element must be placed in any intersection of the block region of $B_{i}$ with block regions of other clause blocks.

First we assume that $\varphi$ is a satisfiable formula and a satisfying variable assignment is given. We need to show that $\left\{\mathcal{P}_{0}, \mathcal{P}_{1}\right\}_{\varphi}$ has a strong embedding. If a variable $x_{j}$ has the value true in the given assignment we open all blocks of negative clauses using $x_{j}$ in the corresponding variable cell; if $x_{j}$ is false we open all blocks of positive clauses using $x_{j}$. Thus no blocks intersect in variable cells any more. If a clause contains more than one true literal, we open all but one connection in its variable cells of true literals. Since the assignment satisfies $\varphi$, we know that each clause block is opened exactly twice in its variable cells and thus forms a valid simple block region. Moreover, we place all shared elements in their home cells so that every block intersection contains an element and the embedding is strong. We call a strong embedding of $\left\{\mathcal{P}_{0}, \mathcal{P}_{1}\right\}_{\varphi}$ with the above properties a canonical embedding.

Now assume that $\left\{\mathcal{P}_{0}, \mathcal{P}_{1}\right\}_{\varphi}$ has a strong embedding. We know that the base grid has its unique embedding and that each block is embedded as a simple region that results from opening the intermediate embedding (with its two E-shapes linked through three variable cells) in exactly two cells. If the embedding is already canonical, we can immediately construct a satisfying variable assignment for $\varphi$ : if a variable cell is crossed by clause blocks in $\mathcal{Q}_{0}$ we set the variable to true, otherwise we set it to false. Since every clause block is connected we know that this assignment satisfies all clauses. 
If the embedding is not canonical we show that it can be transformed into a canonical embedding as follows. In a non-canonical embedding it is possible that two blocks $B_{i}$ and $B_{j}$ intersect in a variable cell $x_{k}$ and have a shared element in their intersection face in the cell of $x_{k}$ rather than in the home cell of that element. This means, on the other hand, that in some shared home cell $\gamma$ of $B_{i}$ and $B_{j}$, say in the upper half, at least one of the two blocks is opened (as there is no more shared element to put into an intersection face). Thus the grid cell $\gamma$ splits the E-shaped block region of one or both blocks in the upper half into two disconnected components, meaning that each opened block crosses at least two variable cells in order to connect both components via the lower half. Hence we can safely split any block that is opened in $\gamma$ in the cell of variable $x_{k}$, re-connect it inside $\gamma$, and place the shared element of $B_{i}$ and $B_{j}$ into its home cell $\gamma$. This removes the block intersection in the cell of $x_{k}$. Once all block crossings within variable cells are removed, the resulting embedding is a canonical embedding and we can derive the corresponding satisfying variable assignment.

\section{Extensions and Conclusion}

We have characterized three main embeddability classes for pairs of partitions, which in fact form a strict hierarchy (see full version [1]), and we have shown NP-completeness of deciding strong embeddability. From a practical point of view the class of strong embeddings is of particular interest: it guarantees that every intersection between block regions is meaningful as it contains at least one element, but, in contrast to full embeddings, it allows multiple disconnected intersection regions between the same two blocks and it allows two blocks to cross.

There are interesting subclasses of strong embeddings that further structure the space between strong and full embeddability. They are discussed in more detail in the full version [1]. In single-intersection strong embeddings we adapt the unique intersection region condition of full embeddings, but still permit that two blocks cross in the embedding. This new class is a true subclass of strong embeddings. It is open whether the corresponding decision problem is still NP-complete since the proof of Theorem 4 is based on the existence of multiple intersection regions between pairs of blocks. In strong grid embeddings, a true subclass of single-intersection strong embeddings, the blocks of $\mathcal{P}_{0}$ and $\mathcal{P}_{1}$ are embedded as horizontal and vertical ribbons, respectively, which intersect in a matrix-like fashion.

It is an interesting direction for future work to generalize our embeddability concepts to $k>2$ partitions. While weak embeddability and its properties extend readily to any number of partitions, it is less obvious how to generalize strong and full embeddability. One possibility is to require the properties in a pairwise sense; otherwise constraints for new types of faces in the contour graph belonging to more than one but less than $k$ block regions might be necessary. On the practical side, future work could be the design of algorithms that find visually appealing simultaneous embeddings of two or more partitions. Finally, if the partitions are clusterings on a graph, one would ideally want to simultaneously draw both the partitions and the underlying graphs.

Acknowledgments. We thank the anonymous reviewers for helpful comments. 


\section{References}

1. Athenstädt, J.C., Hartmann, T., Nöllenburg, M.: Simultaneous embeddability of two partitions. CoRR, abs/1408.6019 (August 2014)

2. Bläsius, T., Kobourov, S.G., Rutter, I.: Simultaneous embedding of planar graphs. In: Tamassia, R. (ed.) Handbook of Graph Drawing and Visualization, ch. 11, pp. 349-381. CRC Press (2013)

3. Brandes, U., Cornelsen, S., Pampel, B., Sallaberry, A.: Blocks of hypergraphs applied to hypergraphs and outerplanarity. In: Iliopoulos, C.S., Smyth, W.F. (eds.) IWOCA 2010. LNCS, vol. 6460, pp. 201-211. Springer, Heidelberg (2011)

4. Brandes, U., Cornelsen, S., Pampel, B., Sallaberry, A.: Path-based supports for hypergraphs. J. Discrete Algorithms 14, 248-261 (2012)

5. Buchin, K., van Kreveld, M., Meijer, H., Speckmann, B., Verbeek, K.: On planar supports for hypergraphs. In: Eppstein, D., Gansner, E.R. (eds.) GD 2009. LNCS, vol. 5849, pp. 345-356. Springer, Heidelberg (2010), see also Tech. Rep. UU-CS-2009-035, Utrecht University (2009)

6. Buja, A., Swayne, D.F., Littman, M.L., Dean, N., Hofmann, H., Chen, L.: Data visualization with multidimensional scaling. J. Comput. Graphical Statistics 17(2), 444-472 (2008)

7. Chaplick, S., Jelínek, V., Kratochvíl, J., Vyskočil, T.: Bend-bounded path intersection graphs: Sausages, noodles, and waffles on a grill. In: Golumbic, M.C., Stern, M., Levy, A., Morgenstern, G. (eds.) WG 2012. LNCS, vol. 7551, pp. 274-285. Springer, Heidelberg (2012)

8. Chow, S.: Generating and Drawing Area-Proportional Euler and Venn Diagrams. PhD thesis, University of Victoria (2007)

9. Collins, C., Penn, G., Carpendale, S.: Bubble sets: Revealing set relations with isocontours over existing visualizations. IEEE TVCG 15(6), 1009-1016 (2009)

10. de Berg, M., Khosravi, A.: Optimal binary space partitions in the plane. In: Thai, M.T., Sahni, S. (eds.) COCOON 2010. LNCS, vol. 6196, pp. 216-225. Springer, Heidelberg (2010)

11. Feng, Q.-W., Cohen, R., Eades, P.: Planarity for clustered graphs. In: Spirakis, P.G. (ed.) ESA 1995. LNCS, vol. 979, pp. 213-226. Springer, Heidelberg (1995)

12. Flower, J., Fish, A., Howse, J.: Euler diagram generation. J. Visual Languages and Computing 19(6), 675-694 (2008)

13. Hopcroft, J., Tarjan, R.: Efficient planarity testing. J. ACM 21(4), 549-568 (1974)

14. Johnson, D.S., Pollak, H.O.: Hypergraph planarity and the complexity of drawing Venn diagrams. J. Graph Theory 11(3), 309-325 (1987)

15. Kaufmann, M., van Kreveld, M., Speckmann, B.: Subdivision drawings of hypergraphs. In: Tollis, I.G., Patrignani, M. (eds.) GD 2008. LNCS, vol. 5417, pp. 396-407. Springer, Heidelberg (2009)

16. Kohonen, T.: Self-Organizing Maps, 3rd edn. Springer (2001)

17. Mäkinen, E.: How to draw a hypergraph. Int. J. Computer Math. 34(3-4), 177-185 (1990)

18. Pach, J., Wenger, R.: Embedding planar graphs at fixed vertex locations. Graphs and Combinatorics 17(4), 717-728 (2001)

19. Simonetto, P., Auber, D., Archambault, D.: Fully automatic visualisation of overlapping sets. Computer Graphics Forum 28(3), 967-974 (2009)

20. S. Wagner and D. Wagner. Comparing Clusterings - An Overview. Tech. Rep. 2006-04, Department of Informatics, Universität Karlsruhe, 2007.

21. Walsh, T.R.: Hypermaps Versus Bipartite Maps. J. Combinatorial Theory Series B 18(2), 155-163 (1975)

22. Zykov, A.A.: Hypergraphs. Russian Mathematical Surveys 29(6), 89-156 (1974) 